\title{
Accepting Medication Therapy Management Recommendations to Add ACEls or ARBs in Diabetes Care
}

\author{
Jason T. Hurwitz, PhD; Amy J. Grizzle, PharmD; Jill Augustine, MPH, PharmD; Rick Rehfeld, BS; \\ Ann Wild, PharmD; and Ivo Abraham, RN, PhD
}

\begin{abstract}
BACKGROUND: National guidelines and initiatives have promoted the use of angiotensin-converting enzyme inhibitors (ACEls) and angiotensin receptor blockers (ARBs) for patients with diabetes. The University of Arizona Medication Management Center (UA-MMC) is contracted by Medicare health plans, pharmacy benefit managers (PBMs), and multiple commercial health insurance plans to provide medication therapy management (MTM) services for plan members. As part of the MTM program, recommendations have been made for those patients who may benefit from the addition of an ACEI/ ARB. Although the intervention benefits and guidelines for using ACEls/ ARBs are clear, real-world evidence is needed to understand and potentially increase uptake of guideline interventions among eligible patients.
\end{abstract}

OBJECTIVES: To (a) identify patient characteristics that predict acceptance of guideline recommendations to add ACEI/ARB medications to diabetic treatment via MTM services and (b) examine how well different case characteristics (i.e., patient age and sex, type and number of recommendation attempts, type of health care plan) predict the odds of adding ACEI/ARB medications to diabetic regimens when recommended through an MTM call center.

METHODS: This was a retrospective analysis of secondary data provided by the UA-MMC. The de-identified national data included adult plan members with diabetes who the UA-MMC recommended adding an ACEI/ARB prescription based on 2012 national guidelines. The UA-MMC made recommendations by either patient letters, patient phone calls, physician faxes, or any combination thereof. We conducted a binary logistic regression analysis to assess the impact of case characteristics on the likelihood of accepting recommendations to add ACEI/ARB medications. The outcome variable was recommendation acceptance (yes/no), defined as new prescription claims for an ACEI/ARB within 120 days following the recommendation. Five predictor variables were assessed: (1) patient's age quartile; (2) method of communicating recommendations (letter, phone call, fax, or some combination thereof); (3) whether recommendations were made once or twice on separate dates; (4) patient's sex; and (5) type of health care plan.

RESULTS: Recommendations were made for 31,495 members of health plans or PBMs that contracted with the UA-MMC. Patients' ages ranged from 19-90 (Mean $=72.01 ; S D=10.21$ ), with females comprising $56 \%$ of the sample. The recommendation to add ACEI/ARB medications was accepted for $14.5 \%(4,559)$ of patients. In most cases $(73 \%)$, recommendations occurred via a letter to patients together with a fax to their providers. The fitted model, containing 3 predictor variables (age quartile, type of contact to communicate the recommendations, and whether recommendation contacts were made twice), was statistically significant, $\chi^{2}$ (10; $\mathrm{N}=31,495)=112.82(P<0.001)$, indicating that the model was able to distinguish between those who did and did not accept UA-MMC's recommendations to add ACEI/ARB medications. The likelihood of recommendation acceptance decreased as patient age increased compared with patients in the first age quartile (ages 19-67; $P \leq 0.005$ at all levels). Compared with sending only a provider fax, patients who received all 3 types of contact (provider fax with patient phone call and letter) were estimated to be 1.34 times more likely (34\% increase) to have recommendation acceptance $(P=0.004 ; 95 \% \mathrm{Cl}=1.10-1.63)$. Similarly, patients who received only letters were also 1.32 times more likely ( $32 \%$ increase) than provider faxes alone to result in recommendation acceptance $(P=0.003 ; 95 \% \mathrm{Cl}=1.10-1.59)$. Patients for whom recommendations were made twice were less likely to have recommendation acceptance than for those contacted once, controlling for all other predictor variables in the model $(P<0.001 ; 0 \mathrm{R}=0.77 ; 95 \%$ $\mathrm{Cl}=0.69-0.86$ ).

CONCLUSIONS: Recommendations to add an ACEI/ARB to diabetic regimens are more likely to be accepted for younger patients and those who receive recommendations through all 3 communication types (provider fax combined with patient phone call and letter) or just letters than provider faxes alone. Further research is needed to understand why prescribers are not accepting MTM recommendations.

J Manag Care Spec Pharm. 2016;22(1):40-48

Copyright $\odot 2016$, Academy of Managed Care Pharmacy. All rights reserved.

\section{What is already known about this subject}

National guidelines and incentives have been developed to encourage adoption of prescribing angiotensin-converting enzyme inhibitors (ACEIs) and angiotensin receptor blockers (ARBs) in diabetic patients to help reduce renal disease progression.

The Centers for Medicare $\&$ Medicaid Services require Medicare Part D sponsors to provide medication therapy management programs to target recommendations such as adding ACEI/ARBs to diabetic regimens.

\section{What this study adds}

Specific patient characteristics and communication methods may lead to higher acceptance rates of adding an ACEI/ARB medication.

Compared with sending only a provider fax, patients who received all 3 types of contact (provider fax with patient phone call and letter) were estimated to be 1.34 times more likely (34\% increase) to have an ACEI/ARB added as recommended.

Patients who received recommendations by patient letters were 1.32 times more likely (32\% increase) to have an ACEI/ARB added than recommendations communicated by provider faxes. 
I $n$ 2012, the prevalence of diabetes in the United States climbed to 22.3 million people, with direct medical costs totaling $\$ 176$ billion. ${ }^{1}$ Approximately 35\% of diabetic patients also had heart disease or stroke (age 35 years or older), while $40 \%$ had been diagnosed with chronic kidney disease., ${ }^{2,3}$ The average lifetime cost of treating diabetes, weighted by age and sex, was $\$ 85,200$, with $53 \%$ of costs used to treat diabetic complications. $^{4}$

Angiotensin-converting enzyme inhibitors (ACEIs) and angiotensin receptor blockers (ARBs) are generally used to help reduce high blood pressure and reduce cardiovascular mortality, including stroke and heart failure..$^{5-7}$ As patients with diabetes are at increased risk for heart disease, stroke, and kidney disease, ACEIs and ARBs are recommended to help reduce renal disease progression, including nephropathy and microalbuminuria. ${ }^{8-12}$ Early treatment with these medications can, therefore, also prevent further renal problems and reduce progression to end-stage renal disease and kidney transplantation. ${ }^{9,13,14}$

National guidelines and initiatives have promoted the use of ACEIs and ARBs for patients with diabetes. At the time of this study, the Centers for Medicare \& Medicaid Services (CMS) recommended that patients with diabetes who have high blood pressure receive an ACEI/ARB. ${ }^{15}$ This guideline was based on recommendations developed and endorsed by the Pharmacy Quality Alliance and endorsed by the National Quality Forum Consensus Standards Committee. ${ }^{11,12,15}$ These guidelines were also consistent with national treatment guidelines published by the American Diabetes Association (ADA), which further recommends the use of ACEIs and ARBs among patients with diabetes who have either hypertension or nephropathy and that ACEIs may be considered for use among these patients who also have cardiovascular disease. ${ }^{11,12}$ While the addition of ACEIs/ARBs continues to be recommended, updated guidelines from the Eighth Joint National Committee (JNC-8) recognize that additional medications, such as calcium channel blockers and thiazide diuretics, may also be used as first-line agents. ${ }^{16}$

While ACEIs and ARBs are promoted as part of the treatment of diabetic patients, they should be avoided or used cautiously in some patient populations. Known side effects of these medications include increased creatinine levels and risk for hyperkalemia. ${ }^{17}$ Patients who experienced previous allergic or adverse reactions to these medications (e.g., angioedema) should avoid their use, as should patients who are pregnant or within childbearing age due to teratogenic effects. ${ }^{18}$ Other contraindications for use of ACEIs/ARBs in this patient population include hypotension, renal artery stenosis, and worsening renal function. ${ }^{19}$ Despite the contraindications, use of ACEIs/ ARBs can benefit the majority of patients with diabetes. Further, several studies have found use of ACEIs in patients with diabetes to be less costly and more effective than without. ${ }^{20-24}$ While diabetes cannot be cured, ACEIs/ARBs can do much to mitigate complications for patients eligible to receive them.
Although the benefits and guidelines for using ACEIs/ARBs were clear at the time of this study, real-world evidence needed to increase uptake among eligible patients remains under way. The purpose of this study is to identify predictors for accepting guideline recommendations to add ACEI/ARB medications to diabetic treatment. Specifically, we assessed how well different case characteristics (i.e., patient age and sex, type and number of recommendation attempts, health care plan) predict the odds of adding ACEI/ARB medications to diabetic regimens per guideline recommendations.

\section{Methods}

\section{Secondary Data and Sample Selection}

Following approval by the University of Arizona institutional review board, we conducted a retrospective analysis of secondary data provided and pre-screened by the University of Arizona Medication Management Center (UA-MMC). To improve health care quality and costs by recommending appropriate use of medications, CMS mandated Medicare Part D sponsors to provide medication therapy management (MTM) programs. ${ }^{25}$ The UA-MMC is contracted by Medicare health plans, pharmacy benefit managers (PBMs), and multiple commercial health insurance plans to provide MTM services for plan members. Part of the program entails follow-up with patients (and/or their providers) whose medical history and/or pharmacy claims trigger alerts of known medication issues, including diabetic patients identified for quality improvement by CMS who may benefit from the addition of an ACEI/ARB. Insights from MTM data offer a rare source of real-word evidence relative to electronic health records and other claims data.

The de-identified national data included adult plan members with diabetes who the UA-MMC recommended adding an ACEI/ARB prescription based on the 2012 ADA guidelines. Recommendations made by the UA-MMC in 2012 and a 120day follow-up period were used to determine whether or not the recommendation was accepted. Outcomes of recommendations made on December 31, 2012, for example, were therefore monitored through April 30, 2013. The 120-day follow up is consistent with other types of UA-MMC alerts, is designed to account for 90-day fills, and is considered reasonable to associate with the recommendations. The UA-MMC communicated recommendations either directly to patients by letter or phone call from a UA-MMC pharmacist, via fax to patients' health care providers, or by some combination thereof. UA-MMC did not have a medical director at the time, but the interventions they conducted were approved by plan sponsors who have medical directors.

Specifically, patients who met the criteria for their health plan's standard MTM program were eligible for a recommendation if they had at least 2 of the following claims in the previous 120 days: alpha-glucosidase inhibitors; biguanides; thiazolidinediones; sulfonylureas; meglitinides; short-acting insulins; 


\begin{tabular}{|c|c|c|}
\hline Variable & Description & Values $^{\mathrm{a}}$ \\
\hline Add ACEI/ARB & Outcome variable: Accepted recommendation to add ACEI/ARB & $\begin{array}{l}0=\text { No } \\
1=\text { Yes }\end{array}$ \\
\hline Age $^{\mathrm{b}}$ & Demographic predictor variable representing the patient's age as of December 31, 2012 & $19-90$ \\
\hline Age quartile & Demographic predictor variable categorizing the sample of patient ages by quartiles & $\begin{array}{l}0=\mathrm{Q} 1: 19-67 \\
1=\mathrm{Q} 2: 68-73 \\
2=\mathrm{Q} 3: 74-79 \\
3=\mathrm{Q} 4: 80-90\end{array}$ \\
\hline Communication method & Predictor variable indicating the mode of patient contact & $\begin{array}{l}0=\text { Fax to physician } \\
1=\text { Letter to patient } \\
2=\text { Phone to patient } \\
3=\text { Phone + letter } \\
4=\text { Fax }+ \text { letter } \\
5=\text { Fax }+ \text { phone } \\
6=\text { Fax }+ \text { phone }+ \text { letter }\end{array}$ \\
\hline Contacted twice & $\begin{array}{l}\text { Predictor variable indicating that the recommendation was attempted twice with a } \\
\text { patient in } 2012\end{array}$ & $\begin{array}{l}0=\text { No } \\
1=\text { Yes }\end{array}$ \\
\hline Female & Demographic predictor variable for patient's sex & $\begin{array}{l}0=\text { Male } \\
1=\text { Female }\end{array}$ \\
\hline Program & Predictor variable indicating the type of health care insurance program & $\begin{array}{l}0=\text { Commercial } \\
1=\text { Medicare }\end{array}$ \\
\hline \multicolumn{3}{|c|}{$\begin{array}{l}\text { aReference groups coded "O" for predictor variables. } \\
\text { "Excluded from model fitting due to data issues identified during preliminary analysis (i.e., linearity in the logit). Recoded and replaced with age quartile (included in this } \\
\text { table). } \\
\text { ACEI = angiotensin-converting enzyme inhibitor; ARB = angiotensin receptor blocker. }\end{array}$} \\
\hline
\end{tabular}

intermediate-, long-acting, or combination insulins; glucagon; incretin mimetic agents; antihyperglycemic hormone analogs; dipeptidyl peptidase-4 (DPP-4) inhibitors; oral dextrose formulations; or diabetic supplies. While all of these patients have diabetes, UA-MMC pharmacists could not verify if they had coexisting conditions (e.g., hypertension, nephropathy) that made patients eligible to receive an ACEI/ARB until after speaking together on the telephone.

Patients who did not meet criteria for their health plan's standard MTM program but were targeted for a separate quality improvement program (expanded MTM) were also eligible for inclusion in this study if they had diabetes and hypertension defined by (a) at least 1 of the above claims filled in the previous 120 days and (b) 1 or more claims for calcium channel blockers or beta blockers (systemic) filled during the same period.

Excluded from analysis were plan members who (a) had a contraindication for receiving ACEIs/ARBs based on concomitant medications (i.e., azathioprine, cyclosporine, lithium, megesterol, midodrine, or potassium-removing resins), (b) previously used an ACEI/ARB, (c) were under age 18, (d) were not continuously enrolled for 120 days following the recommendation, or (e) were women of childbearing age who may be or were actively trying to become pregnant based on claims for prenatal or fertility treatment. Pharmacists who spoke to patients by phone were further able to verify eligibility for the recommendation. The data file provided by UA-MMC for secondary analysis contained only patients who met the inclusion criteria.

\section{Analysis}

We conducted a binary logistic regression analysis of the secondary data to assess the impact of various case characteristics on the likelihood of accepting recommendations to add ACEI/ ARB medications. The outcome variable was recommendation acceptance (yes/no), defined as new prescription claims for an ACEI/ARB within 120 days following the recommendation. If no subsequent claims for an ACEI/ARB appear, then the UA-MMC checks the last date for any prescription claim (other than an ACEI/ARB). If the last claim date is more than 120 days after the recommendation, then the UA-MMC considers the patient continuously enrolled and the recommendation is deemed "not accepted." However, if the last claim date (for a prescription other than an ACEI/ARB) is less than 120 days, then the member has likely disenrolled from the plan and the recommendation outcome is deemed unmeasurable and the case excluded from analysis. UA-MMC adopted this strategy because the disenrollment dates provided by the plans sometimes lagged and were not always dependable.

Table 1 summarizes the 5 predictor variables selected for evaluation from the available data: (1) patient's age quartile; (2) method of communicating recommendations; (3) whether recommendations were made once or twice on separate dates; (4) patient's sex; and (5) type of health care plan. Communication method indicates whether physicians received a fax and whether patients received a phone call, a letter, or both. We used 


\begin{tabular}{|c|c|c|c|}
\hline \multirow{3}{*}{$\begin{array}{l}\text { Sample } \\
\text { Characteristics }\end{array}$} & $\begin{array}{l}\text { ple Ch } \\
\text { ecomm } \\
\text { I/ARB I }\end{array}$ & $\begin{array}{l}\text { ristics a } \\
\text { ions to } \\
\text { tions (I }\end{array}$ & $\begin{array}{l}\text { utcomes } \\
\text { its to Add } \\
\text { 495) }\end{array}$ \\
\hline & \multirow[b]{2}{*}{ n } & \multicolumn{2}{|c|}{ Accept Recommendation } \\
\hline & & No $(\%)$ & Yes (\%) \\
\hline \multicolumn{4}{|l|}{ Patient age quartile } \\
\hline Q1: Ages 19-67 & 8,598 & 83.4 & 16.6 \\
\hline Q2: Ages 68-73 & 8,056 & 85.1 & 14.9 \\
\hline Q3: Ages 74-79 & 7,265 & 86.1 & 13.9 \\
\hline Q4: Ages 80-90 & 7,576 & 87.9 & 12.1 \\
\hline \multicolumn{4}{|c|}{ Communication method } \\
\hline Fax to physician & 1,762 & 87.1 & 12.9 \\
\hline Letter to patient & 1,887 & 83.2 & 16.8 \\
\hline Phone to patient & 152 & 85.5 & 14.5 \\
\hline Phone+letter & 1,423 & 86.1 & 13.9 \\
\hline Fax+letter & 23,101 & 85.5 & 14.5 \\
\hline Fax + phone & 1,750 & 88.1 & 11.9 \\
\hline Fax + phone+letter & 1,420 & 83.2 & 16.8 \\
\hline \multicolumn{4}{|c|}{ Number of recommendation attempts } \\
\hline Once & 28,387 & 85.2 & 14.8 \\
\hline Twice & 3,108 & 88.2 & 11.8 \\
\hline \multicolumn{4}{|l|}{ Sex of patient } \\
\hline Male & 13,989 & 85.7 & 14.3 \\
\hline Female & 17,506 & 85.4 & 14.6 \\
\hline \multicolumn{4}{|l|}{ Health plan ${ }^{\mathrm{a}}$} \\
\hline Commercial & 1,053 & 85.2 & 14.8 \\
\hline Medicare standalone & 16,461 & 86.5 & 13.5 \\
\hline Medicare Advantage & 13,981 & 84.4 & 15.6 \\
\hline Total & 31,495 & 85.5 & 14.5 \\
\hline \multicolumn{4}{|c|}{$\begin{array}{l}\text { aDistinction between Medicare programs are for descriptive purposes. Analysis } \\
\text { compared only commercial versus Medicare. } \\
\text { ACEI=angiotensin-converting enzyme inhibitor; } A R B=\text { angiotensin receptor } \\
\text { blocker. }\end{array}$} \\
\hline
\end{tabular}

interquartile ranges of patient ages (age quartile) because the continuous variable otherwise violated the linearity assumption. Contacted twice accounts for those patients $(n=3,108)$ who had 2 rows of data in the file, each based on 2 different recommendation dates in 2012. This subsample of patients includes those for whom recommendations to add an ACEI/ ARB were not initially accepted but who were later reassessed and deemed still eligible to receive the recommendation. Only those data concerning the most recent of the 2 recommendation dates were retained in the analysis. Potential interactions between age quartile and 2 other predictors were also considered (age quartile $\times$ sex; age quartile $\times$ contacted twice).

Analysis and identification of the fitted model occurred in 3 stages: (1) identify the most parsimonious model to assess the relative importance of each predictor to the overall model fit; (2) interpret results of the fitted model; and (3) evaluate model diagnostics further (e.g., multicollinearity, incomplete information, outliers, cases for which the model fit poorly).

\section{Supplementary Analyses}

While these data exclude women who were or actively trying to become pregnant, the sample does include many women under 60 years old considered to be of childbearing age (based on UA-MMC Clinical Committee consensus). To test for potential bias, a supplementary chi-square analysis was also conducted to evaluate if the proportion of females who accepted the recommendations to add ACEI/ARB medications differed between those under and over age 60. All analyses were conducted using SPSS Version 22 (SPSS Inc., Chicago, IL), and an alpha of 0.05 was used for all tests of significance. ${ }^{26}$

\section{Results}

The data file analyzed in this study, after screening out 99 additional cases with missing data, included a total sample of 31,495 adult members of health plans or PBMs that contracted with the UA-MMC. Patients' ages ranged from 19-90 (Mean $=72.01$; standard deviation $[S D]=10.21$ ), with females comprising $56 \%$ of the sample. Table 2 summarizes additional descriptive statistics for the outcome and predictor variables.

The recommendation to add ACEI/ARB medications was accepted for $14.5 \%(4,559)$ of the 31,495 patients in the sample. The proportions of recommendation acceptance for the standard and expanded MTM groups were 14.2\% and 14.8\%, respectively. In most cases (73\%), recommendations occurred via fax to providers combined with letters to patients. Of the 4,745 cases that involved phoning patients, $14.1 \%$ resulted in accepting the recommendation.

\section{Contributors to Recommendation Acceptance}

We conducted a binary logistic regression analysis to evaluate the influence of multiple case characteristics on the likelihood that UA-MMC's recommendations would be accepted. The full model included 5 categorical predictor variables described in Table 3. Compared with a model with no predictors, the full model with all 5 predictors was statistically significant, $\chi^{2}$ (12; $\mathrm{N}=31,495)=115.61 ; P<0.001$, indicating that the full model could distinguish between plan members who did and did not have UA-MMC's recommendations accepted. The 2 proposed interaction terms (i.e., age quartile $\times$ sex; age quartile $\times$ contacted twice) tested in the follow-up analysis were non-significant.

However, this full model was not a significant improvement compared with a more parsimonious (fitted) model with fewer predictors (change in $\chi^{2}[2]=2.79 ; P=0.248$ ), summarized in Table 3. Re-evaluating the incremental effects of adding each predictor individually to the model indicated that female and health plan variables offered no significant improvement $(P>0.05)$. The fitted model includes 3 predictor variables that have a significant influence on recommendation acceptance: age quartile, method used to communicate the recommendations, and whether recommendation contacts were made twice. 
TABLE 3 Comparison of Coefficients for Full and Parsimonious Models for Predictors of Accepting the Recommendation to Add ACEI/ARB Medications ( $N=31,495$ )

\begin{tabular}{|c|c|c|c|c|c|c|c|c|}
\hline & \multirow[b]{2}{*}{ b } & \multirow[b]{2}{*}{ SE } & \multirow[b]{2}{*}{ Wald } & \multirow[b]{2}{*}{ df } & \multirow[b]{2}{*}{$P$ Value } & \multirow[b]{2}{*}{ OR } & \multicolumn{2}{|c|}{$95 \%$ CI for OR } \\
\hline & & & & & & & Lower & Upper \\
\hline \multicolumn{9}{|l|}{ Full model } \\
\hline Age quartile overall & & & 63.54 & 3 & $<0.001$ & & & \\
\hline Age quartile 2 vs. 1 & -0.133 & 0.044 & 9.20 & 1 & 0.002 & 0.876 & 0.804 & 0.954 \\
\hline Age quartile 3 vs. 1 & -0.210 & 0.046 & 21.14 & 1 & $<0.001$ & 0.810 & 0.741 & 0.886 \\
\hline Age quartile 4 vs. 1 & -0.365 & 0.047 & 60.65 & 1 & $<0.001$ & 0.694 & 0.634 & 0.761 \\
\hline Communication method & & & 24.60 & 6 & $<0.001$ & & & \\
\hline Letter vs. fax & 0.264 & 0.095 & 7.69 & 1 & 0.006 & 1.302 & 1.080 & 1.568 \\
\hline Phone vs. fax & 0.088 & 0.242 & 0.13 & 1 & 0.717 & 1.092 & 0.679 & 1.754 \\
\hline (Phone+letter) vs. fax & 0.052 & 0.105 & 0.25 & 1 & 0.619 & 1.054 & 0.857 & 1.295 \\
\hline$($ Fax + letter) vs. fax & 0.118 & 0.075 & 2.52 & 1 & 0.112 & 1.126 & 0.973 & 1.303 \\
\hline$($ Fax + phone $)$ vs. fax & -0.123 & 0.104 & 1.41 & 1 & 0.235 & 0.884 & 0.722 & 1.083 \\
\hline (Fax + phone + letter) vs. fax & 0.278 & 0.101 & 7.54 & 1 & 0.006 & 1.320 & 1.083 & 1.609 \\
\hline Contacted twice & -0.267 & 0.058 & 20.79 & 1 & $<0.001$ & 0.766 & 0.683 & 0.859 \\
\hline Female & 0.025 & 0.032 & 0.59 & 1 & 0.443 & 1.025 & 0.962 & 1.092 \\
\hline Medicare & 0.137 & 0.093 & 2.20 & 1 & 0.138 & 1.147 & 0.957 & 1.376 \\
\hline Constant & -1.848 & 0.107 & 298.64 & 1 & $<0.001$ & 0.158 & & \\
\hline \multicolumn{9}{|l|}{ Fitted model ${ }^{a}$} \\
\hline Age quartile overall & & & 61.25 & 3 & $<0.001$ & & & \\
\hline Age quartile 2 vs. 1 & -0.119 & 0.043 & 7.80 & 1 & 0.005 & 0.887 & 0.816 & 0.965 \\
\hline Age quartile 3 vs. 1 & -0.197 & 0.045 & 19.32 & 1 & $<0.001$ & 0.822 & 0.753 & 0.897 \\
\hline Age quartile 4 vs. 1 & -0.349 & 0.046 & 58.31 & 1 & $<0.001$ & 0.705 & 0.645 & 0.771 \\
\hline Communication method & & & 25.07 & 6 & $<0.001$ & & & \\
\hline Letter vs. fax & 0.279 & 0.094 & 8.76 & 1 & 0.003 & 1.322 & 1.099 & 1.591 \\
\hline Phone vs. fax & 0.105 & 0.242 & 0.19 & 1 & 0.663 & 1.111 & 0.692 & 1.784 \\
\hline (Phone+letter) vs. fax & 0.068 & 0.105 & 0.42 & 1 & 0.519 & 1.070 & 0.871 & 1.314 \\
\hline$($ Fax + letter $)$ vs. fax & 0.133 & 0.074 & 3.23 & 1 & 0.072 & 1.142 & 0.988 & 1.320 \\
\hline$($ Fax + phone $)$ vs. fax & -0.105 & 0.103 & 1.05 & 1 & 0.305 & 0.900 & 0.736 & 1.101 \\
\hline$($ Fax + phone + letter $)$ vs. fax & 0.291 & 0.101 & 8.36 & 1 & 0.004 & 1.338 & 1.098 & 1.630 \\
\hline Contacted twice & -0.264 & 0.058 & 20.46 & 1 & $<0.001$ & 0.768 & 0.685 & 0.861 \\
\hline Constant & -1.726 & 0.075 & 527.56 & 1 & $<0.001$ & 0.178 & & \\
\hline
\end{tabular}

${ }^{a}$ Model $\chi 2(10, N=31,495)=112.82 ; P<0.001$. Pseudo $R^{2}=0.004$ (Cox E Snell), 0.006 (Nagelkerke).

$A C E I=$ angiotensin-converting enzyme inhibitor; $A R B=$ angiotensin receptor blocker $; b=$ regression coefficient; $C I=$ confidence interval; $d f=$ degrees of freedom; OR $=$ odds ratio; SE = standard error

\section{Extent of Contributions to Recommendation Acceptance}

The fitted model, containing 3 predictor variables, was statistically significant, $\chi^{2}(10 ; \mathrm{N}=31,495)=112.82 ; P<0.001$, indicating that the model was able to distinguish between those who did and did not accept UA-MMC's recommendations to add ACEI/ARB medications. All 3 predictor variables (age quartile, communication method, and contacted twice) made a statistically significant contribution to the model. However, low pseudo $R^{2}$ values suggest the presence of additional potential variables outside those available in this study that may explain more of the variability in outcomes.

The patients' age quartile overall was a statistically significant predictor (Wald $\chi^{2}[3]=61.25 ; P \leq 0.001$ ), holding all other predictors constant. The odds ratios (ORs) suggest that the likelihood of recommendation acceptance decreases as patient age increases (ages of 68-73, 74-79, and 80-90) compared with patients in the first age quartile (ages 19-67); all levels of this variable were significant $(P<0.005)$.

Method of communicating recommendations was also a significant predictor overall of accepting the recommendation to add ACEI/ARB medications (Wald $\chi^{2}[6]=25.07 ; P<0.001$ ), controlling for the other predictor variables. Specifically, compared with only sending a fax to the provider, those who received all 3 communication methods (provider fax combined with patient phone call and letter) were estimated to be 1.34 (34\% increase) times more likely to have recommendation acceptance $(P=0.004 ; 95 \%$ confidence interval $[C I]=1.10$ 1.63). Letters sent to patients without a phone call or provider fax were also 1.32 times more likely ( $32 \%$ increase) than provider faxes alone to result in recommendation acceptance $(P=0.003 ; 95 \% \mathrm{CI}=1.10-1.59)$. 
No other types or combinations of communication methods differed significantly in recommendation acceptance than sending only the fax to providers alone. Compared with recommendations made by provider fax alone, recommendations based on provider fax and patient phone call without the letter were unlikely to influence acceptance $(\mathrm{OR}=0.90 ; 95 \%$ $\mathrm{CI}=0.74-1.10)$, as were recommendations that also combined only letters and faxes $(\mathrm{OR}=1.14 ; 95 \% \mathrm{CI}=0.99-1.32)$. Finally, patients for whom recommendations were made twice were less likely to have recommendation acceptance than for those contacted once, controlling for all other predictor variables in the model $(P<0.001 ; \mathrm{OR}=0.77 ; 95 \% \mathrm{CI}=0.69-0.86)$.

Model diagnostics revealed no collinearity but did indicate some outliers, namely those who received only phone calls, for whom the model might fit poorly. Overall, however, the fitted model appears to be free from dependency or influential outliers.

\section{Comparison of Recommendation Acceptance Between Women Who Were and Were Not of Childbearing Age ( $<60$ Years)}

There was no significant association between the childbearing age of women and whether or not recommendations to add an ACEI/ARB medication were accepted, $\chi^{2}(1 ; \mathrm{N}=17,378)=1.28$; $P=0.258$. Among women of childbearing age $(n=2,212), 14 \%$ added an ACEI/ARB compared with $15 \%$ of women over 60 years old $(n=15,166)$. Pregnancy risk data were unavailable for the remaining 128 females due to discrepant age data, which were excluded from this supplementary subanalysis.

\section{Discussion}

The UA-MMC identified 31,495 health plan members with diabetes who were eligible for adding an ACEI/ARB and who were not otherwise being treated according to this standard of care. Overall, the UA-MMC's recommendations to add an ACEI/ ARB were accepted for $14.5 \%$ of these patients. Proportions of recommendation acceptance ranged across subgroups, from $11.8 \%$ among those who were contacted twice to $16.8 \%$ among communications using just a letter only or all 3 methods combined. The large sample size allowed us to evaluate several potential predictors and the finer differences within them. Further work is required to address the remaining $85.5 \%$ of patients who are not receiving treatment according to the standard of care. These findings suggest ways to improve the effectiveness of MTM recommendations and identify needs for further research.

At the time of this study, MTM recommendations for ACEIs/ ARBs were part of the CMS Star ratings for Medicare Part D health plans. ${ }^{27}$ Depending on the baseline characteristic of a Medicare Part D health plan's diabetes treatment Star rating, a $14.5 \%$ average acceptance rate could be extremely valuable. Based on the 2014 Star measure cut points for diabetes treatment, the difference between a 3-star and a 5-star plan was only an increase of 2 percentage points. ${ }^{28} \mathrm{~A} 14.5 \%$ acceptance rate could move a plan from a 3-star to a 5-star performance level for this measure.

CMS plans to retire the ACEI/ARB recommendation in the 2017 Star ratings based on recent changes in the JNC-8 treatment guidelines. ${ }^{16}$ New evidence suggests that some patients may benefit from first-line treatment with calcium channel blockers and thiazide diuretics. Despite the forthcoming change in Star ratings, this study addresses important aspects of methods implemented by MTM programs to influence prescribing practices. Further research is needed to identify the most effective forms of communication between MTM call centers and prescribers, determine methods for documenting reasons for not accepting recommendations, and ultimately increase the rate of recommendation acceptance. This study sets the stage for answering those important questions, which remain relevant for any recommendations being put forth.

The UA-MMC communicated few recommendations (6\%) only via fax to providers without direct patient contact (letter or phone). This could, in part, be due to invalid phone numbers or addresses supplied by the plans (missing, incorrect, disconnected), patients who were unavailable or did not answer their phones when the UA-MMC called, and patients who declined to speak with the UA-MMC. The influence of provider faxes alone on recommendation acceptance (12.9\%) did not significantly differ from all but 2 types or combinations of communication methods. Nevertheless, provider faxes were used in $89 \%$ of all recommendations, and the acceptance of recommendations ultimately rests upon the prescribers. Although a recent study found that primary care providers were more likely to make changes to ACEI/ARB regimens in diabetic patients compared with nonprimary care providers, further research is needed to understand the influence of prescribers' office characteristics on recommendation acceptance (e.g., centralized refill call center, ancillary staff to support quality improvement activities). ${ }^{29}$

The combination of using all 3 methods to communicate recommendations (provider fax with a patient phone call and letter) appeared to be 1 of 2 methods with the highest acceptance of recommendations ( $16.8 \%$ accepted) but is also the most intensive (e.g., pharmacist time to speak directly with patient, applicable phone and fax charges, printing and mailing fees). Patient letters alone also yielded a 16.8\% acceptance rate, representing the least intensive method that has a significant increase over faxes alone $(P=0.003)$. Patient letters may serve both (a) as a tangible reminder to patients, which they can share and discuss with their providers at their next visit; and (b) to legitimize the phone call, which may otherwise have been dismissed. However, the effect of patient letters combined with only phone calls or only provider faxes did not significantly differ from faxes alone $(P=0.519$ and $P=0.072$, respectively). The relative effect of phoning alone may have been 
difficult to evaluate due to the very small subsample ( $\mathrm{n}=152$, comprising $<0.5 \%$ of recommendations), suggesting an area for further research. Communications involving patient phone calls, however, afford UA-MMC pharmacists an additional opportunity to confirm eligibility apart from relying on the administrative databases.

Further research is also needed to clarify this finding and to compare the cost-effectiveness of other combinations or methods of communication (e.g., patient or provider emails and texts or provider phone calls). There may also be an interplay between certain types of communications and certain types of recommendations. Recent studies found that different types of faxes (informational or prescription) are more effective for some types of MTM recommendations but not others. ${ }^{30,31}$ For example, UA-MMC prescription fax recommendations, which requested provider responses, showed a higher proportion of prescriber approvals for pharmacist recommendations concerning cost savings (58.2\%) than for those concerning guideline adherence (41.4\%) or safety (44.3\%). ${ }^{31} \mathrm{~A}$ recent costeffectiveness simulation compared patient letters to patient phone calls in making UA-MMC's recommendations to add ACEIs/ARBs and/or statin therapies. ${ }^{32}$ Results suggested an overall trade-off between the 2 patient-directed approaches, associating telephone calls with an increased effect and cost relative to the letters. Nevertheless, caution against overloading providers with information must also be taken. Further research may clarify whether additional provider contact may be helpful or counterproductive.

A surprising finding was that being contacted twice was associated with a decreased likelihood of recommendation acceptance. That is, those patients and/or their prescribers who refused the recommendation the first time were more likely persistent in their decisions. One explanation may be that they had medically supported reasons for their decision the first time (e.g., contraindications not otherwise available from the UA-MMC's claims data). However, the acceptance rate among patients whose eligibility was more thoroughly confirmed during phone contact with UA-MMC pharmacists was similar to that of the overall sample population and those cases without phone contact. This suggests that there may be other reasons for declining or ignoring the recommendation, which were unlikely to change upon a second contact. Future research and practice would yield valuable insights during follow-up contacts by documenting reasons for not accepting initial recommendations.

Many reasons may explain why recommendation acceptance decreased among older age groups. One is that many providers may hesitate risking medication changes among patients who have otherwise responded well for a long time without complications. This may be especially true for older patients, who are more likely to have greater health concerns or comorbidities and concomitant medications and thus are at increased risk for drug interactions or other contraindications. Again, additional research on nonacceptance of recommendations could test these hypotheses. Finally, we acknowledge the potential of additional predictors beyond what is available in the current dataset (e.g., patient socioeconomic status, physician specialties and experience, or other communication types) that could be the focus of further real-world outcomes research.

\section{Limitations}

Implications of these results must be considered in light of the study limitations. Low pseudo $R^{2}$ values suggest the presence of additional potential variables outside those available in this study that may explain more of the variability in outcomes. Most importantly, the data do not explain why physicians or patients declined to add the ACEI/ARB medications, allowing us to distinguish between those with medically supported reasons from those without. While the claims data contained enough information to exclude patients based on concomitant medications or women who were pregnant or actively trying to become pregnant, and while phone contacts allowed UA-MMC pharmacists to further confirm eligibility, the data could not fully identify and exclude those with all other possible contraindications. Consequently, results likely underestimate recommendation acceptance and overestimate the proportion of those who declined the recommendations. Future investigations should exclude all cases with known contraindications in advance and follow up with prescribers and patients to determine reasons for declining recommendations. In addition, the 120-day post-recommendation period used to distinguish nonacceptance from disenrollment may be arbitrary (designed to account for 90-day fills, consistent with other UA-MMC alerts, and considered reasonable to associate with the recommendations) but provides a basis for comparison among results of other studies if adopted and applied consistently in future research.

\section{Implications for Clinical Research and Practice}

If many physicians or patients lack medically supported reasons for declining recommendations, then further research is also needed to understand why prescribers are not accepting MTM recommendations. At the time of this study, adding ACEI/ARB medications was a clear guideline. Implementing that guideline was clearly an issue, as fewer than $60 \%$ of diabetic patients with hypertension or renal disease (an indicator for primary rather than preventive treatment) received therapy with an ACEI/ARB. ${ }^{33}$

It has long been recognized that change in prescribing behavior is not a rapid process. On average, it takes 17 years to fully adopt new clinical information generated from randomized clinical trials. ${ }^{34}$ Possibly contributing to this phenomenon is that clinicians are inundated with a multitude of literature and practice guidelines and are challenged to keep pace with 
dissemination. Primary care providers are responsible for managing many conditions, adding to the task of timely guideline implementation. Some research also suggests that primary care providers are also at a disadvantage for quickly adopting new clinical data since they are generally isolated from the academic centers participating in clinical research. Efforts need to be developed to increase awareness and generate evidence-based data to inform changes in prescribing decisions.

\section{Conclusions}

Recommendations are more likely to be accepted for younger patients and those who receive recommendations through multiple interventions (provider fax combined with patient phone call and letter) or just letters than provider faxes alone. While $14.5 \%$ of cases added an ACEI/ARB following the UA-MMC's recommendation, additional evidence is needed to establish the effectiveness of the recommendations. For example, studies can compare the acceptance rates between those who did and did not receive recommendations, compare rates across different types of recommendations, or compare across different MTM contractors for the same recommendation. Further research is also needed to understand the short- and long-term outcomes following recommendations (e.g., adherence, clinical effects, cost-effectiveness) and why prescribers are not accepting MTM recommendations. Results of the current study offer a baseline for further comparison and improving how MTM recommendations are made.

\section{Authors}

JASON T. HURWITZ, PhD, is Assistant Research Scientist; AMY J. GRIZZLE, PharmD, is Assistant Director; RICK REHFELD, BS, is Research Data Analyst; and IVO ABRAHAM, RN, PhD, is Director, Center for Health Outcomes \& PharmacoEconomic Research (HOPE Center), The University of Arizona College of Pharmacy, Tucson. JILL AUGUSTINE, MPH, PharmD, is Doctoral Student, Pharmaceutical Sciences, The University of Arizona College of Pharmacy, Tucson. ANN WILD, PharmD, is Vice President of Operations and Clinical Services, Sinfonía Rx, Tucson, Arizona.

AUTHOR CORRESPONDENCE: Jason T. Hurwitz, PhD, Center for Health Outcomes \& PharmacoEconomic Research (HOPE Center), The University of Arizona College of Pharmacy, 1295 N. Martin Ave., Tucson, AZ 85721. Tel.: 520.626.8889; Fax: 520.626.2023;

E-mail:hurwitz@pharmacy.airzona.edu.
Study design was created by Hurwitz, Grizzle, Rehfeld, and Abraham, with assistance from Augustine and Wild. Wild took the lead in data collection, along with Rehfeld and Augustine. Data interpretation was performed by Hurwitz, Grizzle, Augustine, and Abraham, with assistance from Rehfeld and Wild. The manuscript was written by Hurwitz, Grizzle, and Augustine, with assistance from the other authors, and revised by Hurwitz, Grizzle, Abraham, and Augustine, with assistance from Wild and Rehfeld.

\section{REFERENCES}

1. American Diabetes Association. Economic costs of diabetes in the U.S. in 2012. Diabetes Care. 2013;36(4):1033-46. Available at: http://www.ncbi.nlm. nih.gov/pubmed/23468086. Accessed November 7, 2015.

2. Centers for Disease Control and Prevention. Crude and age-adjusted percentage of people with diabetes aged 35 years or older reporting any heart disease or stroke, United States, 1997-2011. 2012. Available at: http://www.cdc. gov/diabetes/statistics/cvd/fig3.htm. Accessed November 7, 2015.

3. Plantinga LC, Crews DC, Coresh J, et al. Prevalence of chronic kidney disease in US adults with undiagnosed diabetes or prediabetes. Clin J Am Soc Nephrol. 2010;5(4):673-82. Available at: http://www.ncbi.nlm.nih.gov/ pubmed/20338960. Accessed November 7, 2015.

4. Zhuo X, Zhang P, Hoerger TJ. Lifetime direct medical costs of treating type 2 diabetes and diabetic complications. Am J Prev Med. 2013;45(3):253-61. Available at: http://www.ncbi.nlm.nih.gov/pubmed/23953350. Accessed November 7, 2015.

5. McFarlane SI. Role of angiotensin receptor blockers in diabetes: implications of recent clinical trials. Expert Rev Cardiovasc Ther. 2009;7(11):1363-71. Available at: http://www.ncbi.nlm.nih.gov/pubmed/19900019. Accessed November 7, 2015.

6. Strippoli GF, Craig M, Deeks JJ, Schena FP, Craig JC. Effects of angiotensin converting enzyme inhibitors and angiotensin II receptor antagonists on mortality and renal outcomes in diabetic nephropathy: systematic review. BMJ. 2004;329(7470):828. Available at: http://www.ncbi.nlm.nih.gov/ pubmed/15459003. Accessed November 7, 2015.

7. Saha SA, Molnar J, Arora RR. Tissue angiotensin-converting enzyme inhibitors for the prevention of cardiovascular disease in patients with diabetes mellitus without left ventricular systolic dysfunction or clinical evidence of heart failure: a pooled meta-analysis of randomized placebo-controlled clinical trials. Diabetes Obes Metab. 2008;10(1):41-52. Available at: http://www.ncbi.nlm.nih. gov/pubmed/18095949. Accessed November 7, 2015.

8. Rosen AB, Karter AJ, Liu JY, Selby JV, Schneider EC. Use of angiotensinconverting enzyme inhibitors and angiotensin receptor blockers in high-risk clinical and ethnic groups with diabetes. J Gen Intern Med. 2004;19(6):66975. Available at: http://www.ncbi.nlm.nih.gov/pubmed/15209606. Accessed November 7, 2015.

9. Lewis EJ, Hunsicker LG, Clarke WR, et al. Renoprotective effect of the angiotensin-receptor antagonist irbesartan in patients with nephropathy due to type 2 diabetes. N Engl J Med. 2001;345(12):851-60. Available at: http:// www.ncbi.nlm.nih.gov/pubmed/11565517. Accessed November 7, 2014

10. Wong J, Molyneaux L, Constantino M, Twigg SM, Yue DK. Beyond ONTARGET: angiotensin-converting enzyme inhibition and angiotensin II receptor blockade in combination, a lesser evil in some? Diabetes Obes Metab. 2010;12(12):1072-1078. Available at: http://www.ncbi.nlm.nih.gov/ pubmed/20977578. Accessed November 7, 2015.

11. American Diabetes Association. Standards of medical care in diabetes-2012. Diabetes Care. 2012;35 Suppl 1:S11-63. Available at: http://www. ncbi.nlm.nih.gov/pubmed/22187469. Accessed November 7, 2015.

12. American Diabetes Association. Standards of medical care in diabetes-2015. Diabetes Care. 2015;38 Suppl:S1-93. Available at: http://care.diabetesjournals.org/content/38/Supplement_1. Accessed November 7, 2015. 
13. Ruggenenti P, Iliev I, Costa GM, et al. Preventing left ventricular hypertrophy by ACE inhibition in hypertensive patients with type 2 diabetes: a prespecified analysis of the Bergamo Nephrologic Diabetes Complications Trial (BENEDICT). Diabetes Care. 2008;31(8):1629-34. Available at: http:// www.ncbi.nlm.nih.gov/pubmed/18443191. Accessed November 7, 2015.

14. Rennert NJ, Charney P. Preventing cardiovascular disease in diabetes and glucose intolerance: evidence and implications for care. Prim Care. 2003;30(3):569-92. Available at: http://www.ncbi.nlm.nih.gov/pubmed/ 14692202. Accessed November 7, 2015.

15. Centers for Medicare $\&$ Medicaid Services. Medicare health $\&$ drug plan quality and performance ratings: Part C \& D technical notes. Available at: http://www.cms.gov/Medicare/Prescription-Drug-Coverage/Prescription DrugCovGenIn/PerformanceData.html. Accessed November 7, 2015

16. James PA, Oparil S, Carter BL, et al. 2014 evidence-based guideline for the management of high blood pressure in adults: report from the panel members appointed to the Eighth Joint National Committee (JNC 8). JAMA. 2014;311(5):507-20. Available at: http://www.ncbi.nlm.nih. gov/pubmed/24352797. Accessed November 7, 2015.

17. Pappoe LS, Winkelmayer WC. ACE inhibitor and angiotensin II type 1 receptor antagonist therapies in elderly patients with diabetes mellitus: are they underutilized? Drugs Aging. 2010;27(2):87-94. Available at: http://www. ncbi.nlm.nih.gov/pubmed/20104936. Accessed November 7, 2015.

18. Guirguis AB, Shilliday BB, Malone RM, Pignone MP. Avoidance of ACE inhibitors or angiotensin receptor blockers among women of childbearing potential with diabetes. Diabetes Res Clin Pract. 2008;79(1):ell-12. Available at: http://www.ncbi.nlm.nih.gov/pubmed/17716774. Accessed November 7, 2015.

19. The Joint Commission. Specifications manual for Joint Commission National Quality Core (2010B). Available at: http://www.jointcommission. org/specifications_manual_joint_commission_national_quality_core_measures.aspx. Accessed November 7, 2015.

20. Li R, Zhang P, Barker LE, Chowdhury FM, Zhang X. Cost-effectiveness of interventions to prevent and control diabetes mellitus: a systematic review. Diabetes Care. 2010;33(8):1872-94. Available at: http://www.ncbi. nlm.nih.gov/pubmed/20668156. Accessed November 7, 2015.

21. Rippin JD, Barnett AH, Bain SC. Cost-effective strategies in the prevention of diabetic nephropathy. Pharmacoeconomics. 2004;22(1):9-28. Available at: http://www.ncbi.nlm.nih.gov/pubmed/14720079. Accessed November 7, 2015

22. Howard K, White S, Salkeld G, et al. Cost-effectiveness of screening and optimal management for diabetes, hypertension, and chronic kidney disease: a modeled analysis. Value Health. 2010;13(2):196-208. Available at: http:// www.ncbi.nlm.nih.gov/pubmed/19878493. Accessed November 7, 2015.

23. Campbell HM, Boardman KD, Dodd MA, Raisch DW. Pharmacoeconomic analysis of angiotensin-converting enzyme inhibitors in type 2 diabetes: a Markov model. Ann Pharmacother. 2007;41(7):1101-10. Available at: http://www.ncbi.nlm.nih.gov/pubmed/17609233. Accessed November 7, 2015
24. Dong FB, Sorensen SW, Manninen DL, et al. Cost effectiveness of ACE inhibitor treatment for patients with type 1 diabetes mellitus. Pharmacoeconomics. 2004;22(15):1015-27. Availble at: http://www.ncbi.nlm. nih.gov/pubmed/15449965. Accessed September 15, 2014.

25. Centers for Medicare \& Medicaid Services. Requirements for medication therapy management programs (MTMP). Available at: http://www.cms.gov/ Medicare/Prescription-Drug-Coverage/PrescriptionDrugCovContra/MTM. html. Accessed November 7, 2015.

26. IBM Corp. IBM SPSSS Statistics for Windows, Version 22.0. Armonk, NY: IBM Corp.;2013.

27. Academy of Managed Care Pharmacy, American Pharmacists Association. Medicare Star ratings: stakeholder proceedings on community pharmacy and managed care partnerships in quality. J Am Pharm Assoc. 2014;54(3):228-40. Available at: http://www.ncbi.nlm.nih.gov/ pubmed/24770440. Accessed November 7, 2015.

28. Centers for Medicare \& Medicaid Services. Medicare 2014 Part C \& D Star rating technical notes. Available at: http://www.cms.gov/Medicare/ Prescription-Drug-Coverage/PrescriptionDrugCovGenIn/PerformanceData html. Accessed November 7, 2015.

29. Wang J, Surbhi S, Kuhle JW. Receipt of angiotensin-converting enzyme inhibitors or angiotensin II receptor blockers among Medicare beneficiaries with diabetes and hypertension. J Pharm Health Serv Res. 2014;5(1):67-74. Available at: http://www.ncbi.nlm.nih.gov/pubmed/24563667. Accessed November 7, 2015.

30. Cerminara Z, Augustine J, Harrell T, Boesen K. A comparison of the efficacy of two types of faxed medication interventions. Presented at: Academy of Managed Care Pharmacy 26th Annual Meeting \& Expo; April 2014; Tampa, FL.

31. Perera P, Guy M, Sweaney A, Boesen K. Evaluation of prescriber responses to pharmacist recommendations communicated by fax in a medication therapy management program (MTMP). J Manag Care Pharm. 2011;17(5):345-54. Available at: http://www.ncbi.nlm.nih.gov/pubmed/21657804.

32. Olvey E, Guy M, Chang J, Skrepnek G. Cost-effectiveness of medication therapy management in Part D diabetic enrollees. Am J Manag Care. Available at: http://www.ajmc.com/publications/ajpb/2014/ajpb_septemberoctober2014/cost-effectiveness-of-medication-therapy-management-in-partd-diabetic-enrollees/1. Accessed November 7, 2015.

33. Yang Y, Thumula V, Pace PF, Banahan BF 3rd, Wilkin NE, Lobb WB. High-risk diabetic patients in Medicare Part D programs: are they getting the recommended ACEI/ARB therapy? J Gen Intern Med. 2010;25(4):298-304. Available at: http://www.ncbi.nlm.nih.gov/pubmed/20108127. Accessed November 7, 2015.

34. Balas E, Boren S. Managing clinical knowledge for health care improvement. In: Van Bemmel J, McCray A, eds. Yearbook of Medical Informatics 2000: Patient-centered Systems. Stuttgart, Germany: Schattauer; 2000:65-70. 